\title{
Mobilités quotidiennes et localisations : quelle transition énergétique du point de vue des familles?
}

\author{
Joël Meissonnier ${ }^{\mathrm{a}}$ \\ CEREMA, 2 rue de Bruxelles, CS 20275, 59019 Lille Cedex, France
}

\begin{abstract}
Résumé. Deux enquêtes qualitatives par entretiens semi-directifs dans les métropoles françaises de Lille et Lyon explorent les choix de localisation résidentielle en ayant pour focale d'observation les différentes dimensions énergétiques de ces arbitrages. Les enquêtes montrent l'actualité de la thématique énergétique véhiculée par les politiques publiques, les médias ou les experts en climatologie. En France, depuis le Grenelle de l'environnement (2007), la politique publique s'empare de la notion de transition pour signifier que, progressivement et collectivement, il nous faudrait tendre vers des consommations énergétiques plus raisonnées, moins carbonées et moins polluantes. Si les enquêtes accréditent la thèse de la préparation, lente et progressive, d'une transition énergétique dans les représentations sociales, la notion semble moins appropriée pour qualifier les évolutions des pratiques de déplacement observables à l'échelle individuelle et familiale. Si certains ménages adoptent des comportements de mobilité ou des arbitrages de localisation relativement radicaux et allant dans le sens d'une mobilité durable, l'essentiel des ménages déploie des formes d'ajustement qui permettent de « faire le dos rond » pour faire face aux évolutions des conditions de prix de l'énergie. En somme, à l'échelle des arbitrages familiaux de localisation, les tensions sur les prix de l'énergie produisent moins une progressive transition qu'elles ne creusent un fossé entre changement de paradigme et continuité conservatrice.
\end{abstract}

\begin{abstract}
The author contributed to two qualitative surveys with semi-directed interviews in the french cities of Lille and Lyon. He explores residential location choices by taking into account energy consumption. Public policies, media and climate experts urge people to be more and more conscious of the energetic consequences of their choices. In France, since the Grenelle of the Environment (2007), the public policy uses the concept of transition. Gradually and collectively, we should strive towards a more rational energy consumption with less carbon emission. If some households adopt radical mobility behavior or localization choices in accordance with sustainable mobility principles, some other households deploys some forms of adjustment that allow to deal with changes according to energy prices. In short, as far as family location choices are concerned, pressures on energy prices produce less a gradual transition than they dig a gap between the paradigm change and the conservative continuity.
\end{abstract}

\footnotetext{
${ }^{a}$ e-mail : joel.meissonnier@cerema.fr
}

This is an Open Access article distributed under the terms of the Creative Commons Attribution License 4.0, which permits unrestricted use, distribution, and reproduction in any medium, provided the original work is properly cited. 


\section{Du contexte énergétique global aux comportements de déplacement routiniers individuels : d'illusoires transitions?}

La notion de transition énergétique est née en Allemagne et en Autriche dans les années 1980, lorsque se dessine l'optique d'un abandon de la dépendance au pétrole et à l'atome dans ces pays. Elle apparaîtra plus tardivement en France et sera élevée au rang d'objectif politique dans le cadre du Grenelle de l'Environnement ainsi qu'avec la mise en place des Schémas régionaux climat-air-énergie et des Plans « Climat». La notion traduit l'objectif politique de passer progressivement d'énergies dites carbonées (pétrole, gaz naturel, charbon) ou très technologiques et centralisées (nucléaire...) à des énergies propres, sûres et décentralisées.

Pour plusieurs auteurs s'essayant à une prospective énergétique à l'échelle macro-sociale, cette idée de progressivité des changements est critiquable. Ivan Illitch [1] le disait en ouverture de son ouvrage fondateur Énergie et équité, nos sociétés se fourvoient ; on confond bien-être et énergie


appellent à un électrochoc des plus révolutionnaires. Nous serions engagés dans un irrémédiable processus «d'inversion des raretés » [2] menant à un changement de paradigme sociétal radical, tant économique que politique, qui nous contraint à dépasser la modernité [3,4]. Ces travaux en appellent à des changements qui, étant donné l'échelle d'observation macro-sociale et planétaire qu'ils considèrent, font figure d'appel à une révolution des modes de consommation, d'action, de décision, contradictoire avec l'évolution à laquelle la notion de transition énergétique semble appeler. Notre propos consistera à montrer que la progressivité associée à la notion de transition énergétique est tout aussi illusoire à l'échelle plus micro-sociale, celle du ménage et de ses pratiques quotidiennes.

À cette échelle, il n'y aurait pas d'autre issue qu'un changement des comportements individuels car «les acteurs politiques ne peuvent qu'accompagner le mouvement» [5] et non l'inverser. Manuel de transition en main [6], il reviendrait à chaque citoyen de minimiser son «bilan carbone » par une évolution pas à pas des comportements de consommation, de mobilité, par des relocalisations de résidence ou d'activités, par des circuits de distribution courts et des actions citoyennes. C'est mal connaître la réalité des contraintes de mobilité quotidienne qui s'exercent sur nos contemporains. Si par le passé, l'emploi a pu être légitimement refusé lorsqu'il était trop éloigné du domicile, cette légitimité se perd aussi vite que l'emploi se raréfie. Si la «pendularité » quotidienne sur des distances domicile-travail ou domicile-étude toujours plus importantes a pu être un handicap, elle est désormais la norme. Elle passe pour une injonction qui peut s'analyser comme une idéologie mobilitaire [7]. C'est ainsi que l'on s'est engagé dans un processus de périurbanisation reposant sur le règne quasi-absolu de l'automobile, en complète contradiction avec les injonctions à une transition énergétique.

D'un côté l'injonction moderne à la mobilité, de l'autre l'injonction alternative à la transition énergétique. Au milieu, le citoyen devrait seul arbitrer le paradoxe. Comment fait-il ? Comment les citoyens s'emparent-ils, ou non, des messages injonctifs issus des lobbys énergétiques, des militants associatifs, des collectivités, des entreprises ou du législateur ? Il convient d'examiner la transition énergétique «par le bas » [8] en s'intéressant aux acteurs ordinaires, en se mettant au niveau des hommes qui la font ou la défont.

En investiguant les arbitrages de localisation résidentielle et les arbitrages de mobilité d'un point de vue plus compréhensif, on montrera comment les familles s'accommodent des paradoxes.

A À l'échelle des valeurs on assisterait à une réelle et progressive transition culturelle (diffusion des normes de préservation de l'écosystème par exemple).

A Mais à l'échelle des pratiques on assisterait davantage à des prises de position sans transition sur le plan énergétique (la rupture ou la continuité moyennant quelques ajustements à la marge).

b Groupe d'experts intergouvernemental sur l'évolution du climat. 
Tableau 1. Descriptif des familles enquêtées.

\begin{tabular}{|c|c|c|c|}
\hline & Lieu & \multicolumn{2}{|r|}{$\begin{array}{l}\text { Enquêtés selon l'enquête : } \\
\text { A : Accédants à la propriété } \\
\text { S : Salariés travaillant en zone d'activité périphérique. }\end{array}$} \\
\hline Donia et Stéphane & Lyon & A & $\begin{array}{l}\text { Stéphane : chef maçon BTP. } \\
\text { Donia : études de psychologie, n'a pas le permis. } \\
2 \text { jeunes enfants. } \\
\text { Habitent à Vaux-en-Velin. } \\
\text { Achètent un terrain à Villebois. }\end{array}$ \\
\hline Leila et Mohammed & Lille & A & $\begin{array}{l}\text { La trentaine } \\
\text { Plusieurs enfants en bas âge. } \\
\text { En location à Villeneuve d'Ascq } \\
\text { Font construire une maison écologique à Lesquin. } \\
\text { Mohammed : ingénieur en informatique, va au travail à pied. } \\
\text { Leila : sans emploi actuellement. }\end{array}$ \\
\hline Clémentine et Ismaël & Lyon & A & $\begin{array}{l}\text { Habitent : un T4 à Vaise } \\
\text { Clémentine : Gestionnaire immobilier } \\
\text { Ismaël : technicien du son à Vaise } \\
\text { Un seul véhicule } \\
\text { Pas d'enfant }\end{array}$ \\
\hline Chloë et Cédric & Lille & A & $\begin{array}{l}\text { La trentaine } \\
\text { En couple depuis } 9 \text { ans mais vivent encore chez leurs parents. } \\
\text { Cédric : à La Bassée } \\
\text { Chloë : à Sainghain-en-Weppes } \\
\text { Cédric : entraîneur sportif en CDD à Fâches-Thumesnil. } \\
\text { Chloë : assistante à l'université depuis } 2 \text { ans. } \\
\text { achèteront une maison à Lomme } \\
\text { Pas d'enfant. }\end{array}$ \\
\hline Christiane et Jacques & Lyon & A & $\begin{array}{l}\text { intermittents du spectacle } \\
\text { la cinquantaine } \\
\text { trois enfants de } 20,18 \text { et } 14 \text { ans. } \\
\text { habitent à Caluire } \\
\text { affichent une franche hostilité à l'omniprésence de la voiture. }\end{array}$ \\
\hline Pascal et Béatrice & Lyon & A & $\begin{array}{l}\text { jeune couple de moins de } 30 \text { ans } \\
\text { plusieurs séjours à l'étranger } \\
\text { un départ pour un an au Canada en perspective. } \\
\text { vivent dans un } 45 \mathrm{~m}^{2} \text { à Villeurbanne. } \\
\text { Béatrice : a vécu à Croix-Rousse, travaille dans l'informatique. } \\
\text { Pascal : cuisinier, n'a pas le permis. Béatrice travaille dans l'informatique. }\end{array}$ \\
\hline Domitille et Serge & Lille & & $\begin{array}{l}\text { habitent à Hem dans une villa. } \\
\text { Domitille : } 40 \text { ans, cadre dans une entreprise de bricolage à Templemars. } \\
\text { Serge : un peu plus âgé, travaille à Roubaix, chef d'entreprise. } \\
\text { deux jeunes enfants. }\end{array}$ \\
\hline Philippine et Anatole & Lille & S & $\begin{array}{l}\text { Philippine : travaille dans la communciation à Templemars. } \\
\text { Anatole : travaille dans la grande distribution, à Flers. } \\
\text { Ont environ } 45 \text { ans. } \\
\text { Habitent à Villeneuve d'Ascq. } \\
\text { Philippine a } 2 \text { enfants (adolescents) d'une précédente union. }\end{array}$ \\
\hline Céline et Yann & Lille & A & $\begin{array}{l}\text { Céline et Yann : en location à Lambersart } \\
\text { Accèdent à la propriété à Erquinghem } \\
\text { travaillent tous les deux dans la banque. } \\
30 \text { ans et vont bientôt avoir un enfant. }\end{array}$ \\
\hline Frédéric & Lille & A & $\begin{array}{l}\text { Frédéric est célibataire. } \\
\text { approche la quarantaine. } \\
\text { vient d'acheter à Croix } \\
\text { travaille à Roubaix dans l'informatique }\end{array}$ \\
\hline
\end{tabular}




\section{SHS Web of Conferences}

Tableau 1. Continued.

\begin{tabular}{|c|c|c|c|}
\hline Zoë et Oscar & Lille & $\mathrm{S}$ & $\begin{array}{l}\text { habitent à Fretin } \\
\text { approchent de la quarantaine. } \\
\text { Zoë : analyste « base de données » à Templemars } \\
\text { Oscar : travaille sur des chantiers et a de longues périodes d'absence } \\
\text { régulièrement. } \\
\text { jumeaux de } 4 \text { ans. } \\
\text { Pendant } 3 \text { ans Zoë travaillait à Tours et Oscar à Roubaix (double-résidence). }\end{array}$ \\
\hline Jules et Lucile & Lille & $\mathrm{S}$ & $\begin{array}{l}\text { habitent à Lille. } \\
\text { Trentenaires, propriétaires. } \\
\text { Jules : cadre dans une entreprise de Templemars. } \\
\text { Lucile : ingénieur dans un bureau de conseil pour l'environnement. } \\
\text { une fille } \\
\text { ont délibérément choisi la localisation de leur logement pour avoir } \\
\text { facilement accès au centre-ville (où travaille Lucile) en métro. }\end{array}$ \\
\hline Francine et Gérard & Lille & $\mathrm{S}$ & $\begin{array}{l}\text { Trentenaires } \\
\text { habitent Bersée } \\
\text { ont été locataires dans ce bourg avant de faire construire } \\
\text { Francine : agent de maîtrise à Templemars } \\
\text { Gérard : chauffeur routier (rentre le soir à domicile) } \\
\text { Un bébé. }\end{array}$ \\
\hline Françoise et Patrick & Lille & A & $\begin{array}{l}\text { environ } 40 \text { ans } \\
\text { actuellement en sous-location informelle à Wattrelos. } \\
\text { Françoise : chargée de recouvrement chez un huissier à Lille. } \\
\text { Patrick : électricien dans une entreprise de Wattrelos. } \\
\text { un enfant de } 4 \text { ans. }\end{array}$ \\
\hline Stéphanie & Lille & A & $\begin{array}{l}\text { célibataire } \\
\text { trentenaire } \\
\text { originaire de Lens et vit actuellement à Valencienne. } \\
\text { prestataire d'EDF } \\
\text { accède à la propriété à Tourcoing. }\end{array}$ \\
\hline Jean-Charles et Marie & Lille & A & $\begin{array}{l}\text { habitent La Madeleine } \\
\text { accèdent à la propriété à Santes } \\
\text { pas d'enfant } \\
\text { Jean-Charles : travaille à Dunkerque et à Valenciennes } \\
\text { (sécurité sur chantiers). } \\
\text { Marie : agent de voyages à Lille }\end{array}$ \\
\hline Hubert et Fabienne & Lille & $\mathrm{S}$ & $\begin{array}{l}\text { Hubert : dessinateur-projeteur, actuellement à Templemars. } \\
\text { Fabienne : travaille dans une école de commerce (à Euralille) et s'y } \\
\text { rend en bus. } \\
\text { Ont une jeune enfant : } 40 \mathrm{~h} / \mathrm{sem} \text { chez sa nounou. } \\
\text { habitent le Vieux Lille en location. } \\
\text { cherchent à acheter. }\end{array}$ \\
\hline Brigitte et Sophiane & Lille & A & $\begin{array}{l}\text { Brigitte : documentaliste à l'université. } \\
\text { Sophiane : né au Maroc, enseignant en lycée. } \\
\text { locataires à La Madeleine. } \\
\text { Ont quarante ans } \\
\text { veulent accéder à la propriété (perspetive enfant) } \\
\text { Écolo-économe, sont très sensibles tant aux prix qu'aux conséquences } \\
\text { environnementales de leurs choix. }\end{array}$ \\
\hline Junie et Fabien & Lille & $\mathrm{S}$ & $\begin{array}{l}\text { habitent à Marcq-en-Baroeul (en maison) } \\
\text { petite quarantaine } \\
\text { Un enfant de } 4 \text { ans } \\
\text { Junie : chef de service RH au siège d'une entreprise à Templemars. } \\
\text { Fabien : travaillait à Croix pour une entreprise de confection textile avant } \\
\text { de prendre un poste de cadre au siège d'une entreprise de bricolage. } \\
\text { se disent « par principe très sensibles à tout ce qui est économie d'énergie ». }\end{array}$ \\
\hline
\end{tabular}


Tableau 1. Continued.

\begin{tabular}{|l|l|l|l|}
\hline Tanguy et Suzanne & Lille & S & $\begin{array}{l}\text { habitent dans une ferme en Belgique en direction de Bruxelles } \\
\text { approchent de la cinquantaine } \\
\text { Suzanne : tient une ferme pédagogique } \\
\text { Tanguy : cadre (économiste) au siège d'une entreprise de Templemars. } \\
\text { ont ensemble une fille de 9 ans et Suzanne a eu 2 garçons qui ont désormais } \\
\text { quitté le foyer. } \\
\text { Tanguy se dit «plutôt écolo ». }\end{array}$ \\
\hline Clara et Florent & Lille & A & $\begin{array}{l}\text { Trentenaires } \\
\text { A'ont pas d'enfant } \\
\text { accèdent à la propriété à Bois Blanc } \\
\text { sont sensibles aux problèmes environnementaux. } \\
\text { Florent : chargé de mission en habitat durable (Bailleul). } \\
\text { Clara : libraire (Armentières). } \\
\text { Font du covoiturage quotidien pour se rendre sur leurs lieux de travail } \\
\text { respectifs. }\end{array}$ \\
\hline Manuel et Lola & Lille & S & $\begin{array}{l}\text { La quarantaine } \\
\text { Manuel : acheteur dans une entreprise de Templemars. } \\
\text { Lola : a une affaire (café) et tient à sa clientèle à Cambrai. } \\
\text { pas d'enfant } \\
\text { fait du covoiturage régulièrement pour des raisons financières. }\end{array}$ \\
\hline Emilie et Serge & Lille & Lille & $\begin{array}{l}\text { Aabitent La Madeleine. } \\
\text { Amilie : cadre pour une entreprise textile à Roubaix } \\
\text { Serge : consultant, à Lomme. } \\
\text { La petite quarantaine } \\
\text { n'ont pas d'enfant }\end{array}$ \\
\hline Sandrine et Alain & Lille & $\begin{array}{l}\text { La quarantaine } \\
\text { Alain : chauffeur bus (dépôt à Marcq-en-Baroeul). } \\
\text { Sandrine : femme de ménage dans l'hostellerie à Villeneuve d'Ascq } \\
\text { (actuellement en congé parental). } \\
\text { Aabitent à Wattrelos. } \\
\text { le troisième enfant dort sur le pallier, dans le couloir. }\end{array}$ \\
\hline $\begin{array}{l}\text { en location à Marcq-en-Baroeul mais accèdent à la propriété à Marcq bientôt. } \\
\text { Benôt : directeur d'un centre social pour handicapés à Roubaix } \\
\text { auraient préférer déménager dans le périurbain mais ont réalisé que ça } \\
\text { risquait de ne pas être « pratique ». } \\
\text { Corine : éducatrice et travaille dans le centre de Lille. } \\
\text { deux enfants, chacun leur véhicule } \\
\text { environ 35 ans. }\end{array}$ \\
\hline
\end{tabular}

\section{Méthodologie}

Deux enquêtes qualitatives par entretiens semi-directifs dans les métropoles françaises de Lille et Lyon permettent de comprendre les choix résidentiels tant dans leurs dimensions énergétiques intrinsèques (qualité des isolants, type de chauffage, de vitrages...) que dans leurs dimensions énergétiques relatives (modalités d'accès et proximités relatives des destinations de navettes quotidiennes).

Les populations enquêtées ont été choisies pour être potentiellement impactées par l'évolution des prix de l'énergie. La première enquête (une quarantaine d'entretiens) s'est intéressée aux candidats à l'accession à la propriété et, dans la mesure du possible, nous nous sommes efforcés d'interroger des familles de petits-moyens représentants d'une «classe populaire salariée stable » [9]. La seconde (une trentaine d'entretiens) s'intéresse aux salariés travaillant dans des zones d'activités situées en périphérie du tissu urbain, souvent moins bien desservies par les transports collectifs. Chacune de ces enquêtes, a 
eu pour terrains les métropoles de Lille et Lyon. Elles ont été réalisées par une équipe de six sociologuesanthropologues ${ }^{\mathrm{c}}$.

La méthodologie visait d'abord à interroger les arbitrages de localisation résidentielle. Par conséquent, les sociologues ont veillé à ne pas introduire artificiellement la dimension énergétique, à ne pas induire de jugement chez ceux de nos interlocuteurs qui s'exprimaient sur leurs choix modaux et leurs choix de localisation.

Cette contribution se focalise donc sur les dimensions énergétiques de la mobilité quotidienne produite par une localisation résidentielle et des destinations quotidiennes données. En France, contrairement aux dimensions énergétiques intrinsèques au bâti, elles ne font l'objet d'aucune politique publique particulière [10] : pas de crédit d'impôts pour promouvoir des initiatives vertueuses, pas de diagnostic de performance énergétique rendu obligatoires par la loi. L'impact énergétique des mobilités produites par nos choix de localisation seraient-ils méconnus ? négligés ? y sommes-nous indifférents ? Qu'en disent les familles?

\section{Quelle transition énergétique du point de vue des familles ?}

À l'échelle d'observation familiale, on identifie deux grands postes de dépense énergétique : mobilités quotidiennes et chauffage $^{\mathrm{d}}$. Le moment du choix résidentiel est déterminant pour chacun de ces deux postes de dépense, parfois pour de longues années, même si l'un et l'autre ne semblent pas être mis en balance dans une perspective strictement énergétique par les familles. La dimension financière prévaut de même que la perspective de pouvoir agir sur ces postes de dépense. Xavier Desjardins et Lucile Mettetal [11] ont bien montré que « les habitants ont le sentiment de pouvoir maîtriser leurs consommations énergétiques » intrinsèques au logement et disent au contraire, que « dans le domaine de la mobilité, la consommation énergétique leur semble incompressible », d'autant plus fréquemment qu'ils vivent dans le périurbain.

La recherche Transénergy ${ }^{\mathrm{e}}$ a eu pour ambition d'étudier l'adéquation entre choix de localisation et niveaux de consommation énergétique dans la perspective d'une transition allant dans le sens d'une dépense énergétique plus mesurée. Nous nous focalisons sur les consommations énergétiques de mobilité. Examinons ce que les propos des familles interrogées nous permettent d'en dire.

\subsection{Vers une transition des représentations?}

L'intégration d'enjeux planétaires tels que la déplétion des ressources énergétiques fossiles et la tendance haussière des prix (de carburant, de gaz) qu'elle entraîne mécaniquement se diffusent indéniablement dans les représentations sociales de l'énergie des personnes interrogées. Ces enjeux relatifs à l'énergie semblent entrer dans le lot des évidences communes, dans le socle de la connaissance ordinaire. Les représentations sociales de l'énergie apparaissent, souvent en filigrane, au fil du discours, dans leurs conséquences davantage que dans leurs causes. Lorsqu'on y est attentif, on peut entendre les

\footnotetext{
${ }^{c}$ Cécile Vignal, Blandine Mortain, Nathalie Ortar, Félicie Drouilleau, Hélène Ducourant, Joël Meissonnier.

d Ce ne sont pas les seuls - mentionnons à titre d'exemple la cuisson, l'éclairage ou l'usage d'appareils électro-ménagers - mais il s'agit souvent des deux principaux postes de dépense dans les budgets des familles.

${ }^{\mathrm{e}}$ Recherche ANR intitulée «Les stratégies d'adaptation des ménages et des entreprises face à la transition énergétique : une comparaison entre les métropoles de Lille et de Lyon ».
} 
propos qui suivent, sans même que le/la sociologue ne les suscite :

Donia $^{\mathrm{f}} \mathrm{s}$ 'est renseignée sur Internet et déclare faire une différence entre une source d'énergie comme « le bois » et d'autres énergies dont les ressources sont limitées : «ça ne va pas s'arrêter comme le gazole».

À leur mesure, certaines personnes anticipent l'avenir énergétique et les politiques publiques qui lui seront associées.

Sophiane dit : « Je pense que les choses vont évoluer dans le futur. [L'État va finir par] imposer une sorte de taxe en fonction de la consommation d'énergie. Donc voilà. . . il faut s'y préparer ».

Certains interlocuteurs ayant entendu parler des dynamiques de métropolisation en viennent à se projeter dans un avenir où la pression démographique serait telle qu'ils risquent de ne plus avoir les ressources suffisantes pour vivre à proximité suffisante de leurs lieux de travail voire pour s'offrir la consommation mensuelle de carburant pour s'y rendre.

Clémentine et son conjoint ont une voiture mais ils veulent «éviter de la prendre » pour les déplacements quotidiens, notamment « pour le prix de l'essence » outre le fait que c'est « un stress supplémentaire ». Clémentine déclare: « on a réfléchi en se disant : bon, eh bien, l'essence n'est pas près de diminuer, on va être de plus en plus [nombreux]... On a lu des études. À Lyon, ils prévoient que l'on soit, en 2030, plus de 3 millions... ». Et Clémentine de conclure : « Pour aller au boulot, on ne veut pas prendre la voiture, c'est sûr », ce qui les amène à rechercher du côté de Vaise.

Concrètement, les représentations sociales relatives à l'énergie, au côté d'autres représentations relatives à la banalité de l'automobile, contribuent à faire descendre la voiture particulière du piédestal où l'époque moderne l'avait juchée. Des propos reléguant la voiture au rang de moyen de transport dépassé sont de plus en plus largement partagés - tout particulièrement au sein de la classe moyenne active et diplômée - sans d'ailleurs qu'un argument énergétique n'ait besoin d'être explicitement invoqué. Ainsi, la pénibilité des encombrements routiers pousse certaines personnes interrogées à établir explicitement des équivalences monétaires, c'est-à-dire à juger qu'une qualité de vie mérite largement un sacrifice financier.

Junie n'aime pas ses navettes quotidiennes, en voiture, qu'elle passe dans les embouteillages sur l'A1 et qu'elle vit comme une contrariété. Elle dit : «Demain, si on me propose le même boulot où je peux aller en bus ou à pied, c'est. . . [émerveillement] je signe tout de suite, même avec $20 \%$ de salaire en moins !»

«L'erreur de la petite maison de village : 640 euros de transports par mois $»^{\mathrm{g}}$, pouvait-on lire en titre d'un article de la presse en ligne. L'arbitrage complexe entre choix de localisation résidentielle et mobilité quotidienne devient objet de débat. Pour l'alimenter, certaines représentations sociales entrent indéniablement en consonance avec des pratiques de déplacement moins énergivores et allant dans le sens d'une transition énergétique. Mais si on en questionne l'émergence dans une perspective compréhensive, il apparaît assez vite qu'elles n'ont rien d'une inébranlable armature idéologique. Elles sont au mieux reliées à une connaissance de l'actualité énergétique planétaire ; elles naissent surtout d'une prise de conscience des évolutions des prix du carburant. Le plus souvent, ces représentations sociales sont sans rapport direct avec l'ambition politique de transition énergétique décrite plus haut. Elles sont bien davantage le fruit de préférences individuelles pragmatiques traduisant une aspiration à la réduction des tensions qui pèsent sur les budgets et les emplois du temps familiaux.

\footnotetext{
f Pour un descriptif de l'ensemble des personnes interrogées, on se reportera au tableau situé en annexe ainsi qu'aux cartes des toponymes employés.

g rue89.nouvelobs.com/2014/02/16/lerreur-petite-maison-village-640-euros-gasoil-mois-249978.
} 


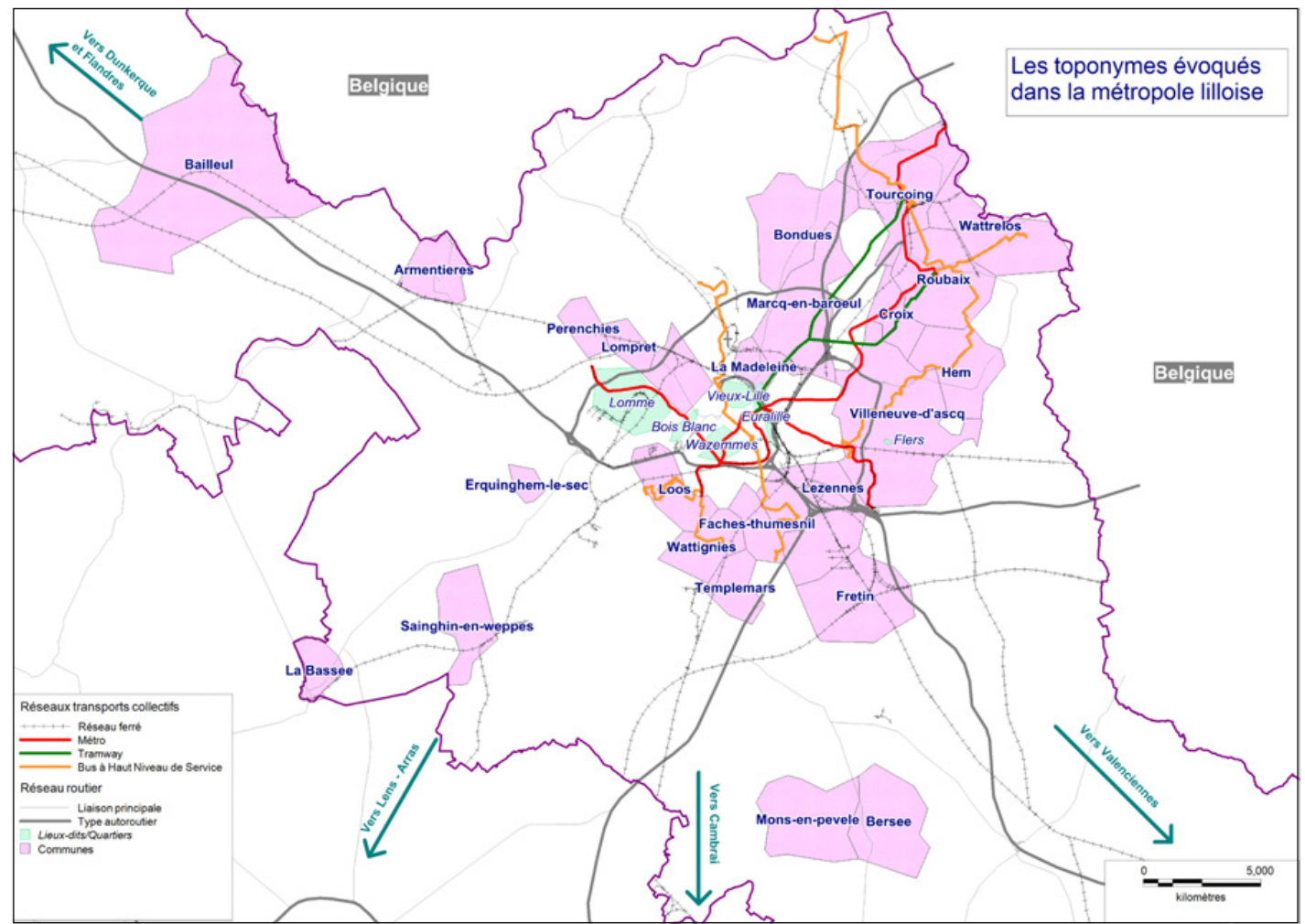

Figure 1. Métropole lilloise : les toponymes employés.

\subsection{Du côté des pratiques : trois catégories distinctes de comportements éco-compatibles font-elles une transition?}

\subsubsection{La bonne volonté écolo-militante}

Les personnes affichant leurs soucis de préserver l'environnement ou de lutter contre le gaspillage énergétique éprouvent la fragilité de leurs convictions et les potentielles contradictions que comporte leur mode de vie à chaque occasion de mobilité. Ces contradictions sont d'autant plus vives que la famille vit dans le lointain périurbain, pour des raisons explicitement énergétiques. Des tactiques de mise en cohérence des valeurs et des pratiques sont alors imaginées. Il s'agit en effet de réduire la dissonance cognitive [12]. C'est ainsi que l'on désigne le malaise psychologique que ressentent plusieurs de nos interlocuteurs dont la sensibilité environnementale entre en contradiction avec des pratiques de mobilité énergivores. Stéphane Labranche [13] considère qu'il n'est pas abusif de qualifier de schizophrénie cette tension en situation d'arbitrage ${ }^{\mathrm{h}}$.

Pour plusieurs personnes revendiquant une sensibilité écologique, une façon de réduire cette dissonance passe par le rapprochement des localisations relatives (domicile et travail-s). S'il est tout à fait anecdotique, l'exemple le plus marquant est celui de Brigitte. En accession à la propriété, elle a

\footnotetext{
$\mathrm{h}$ «Le « schizophrène écologique», comme n’importe quel autre individu, fait constamment une évaluation des différents coûts et avantages, économiques et autres, mais de plus, il se sent coupable lorsqu'il ne traduit pas ses valeurs en actes ».
} 


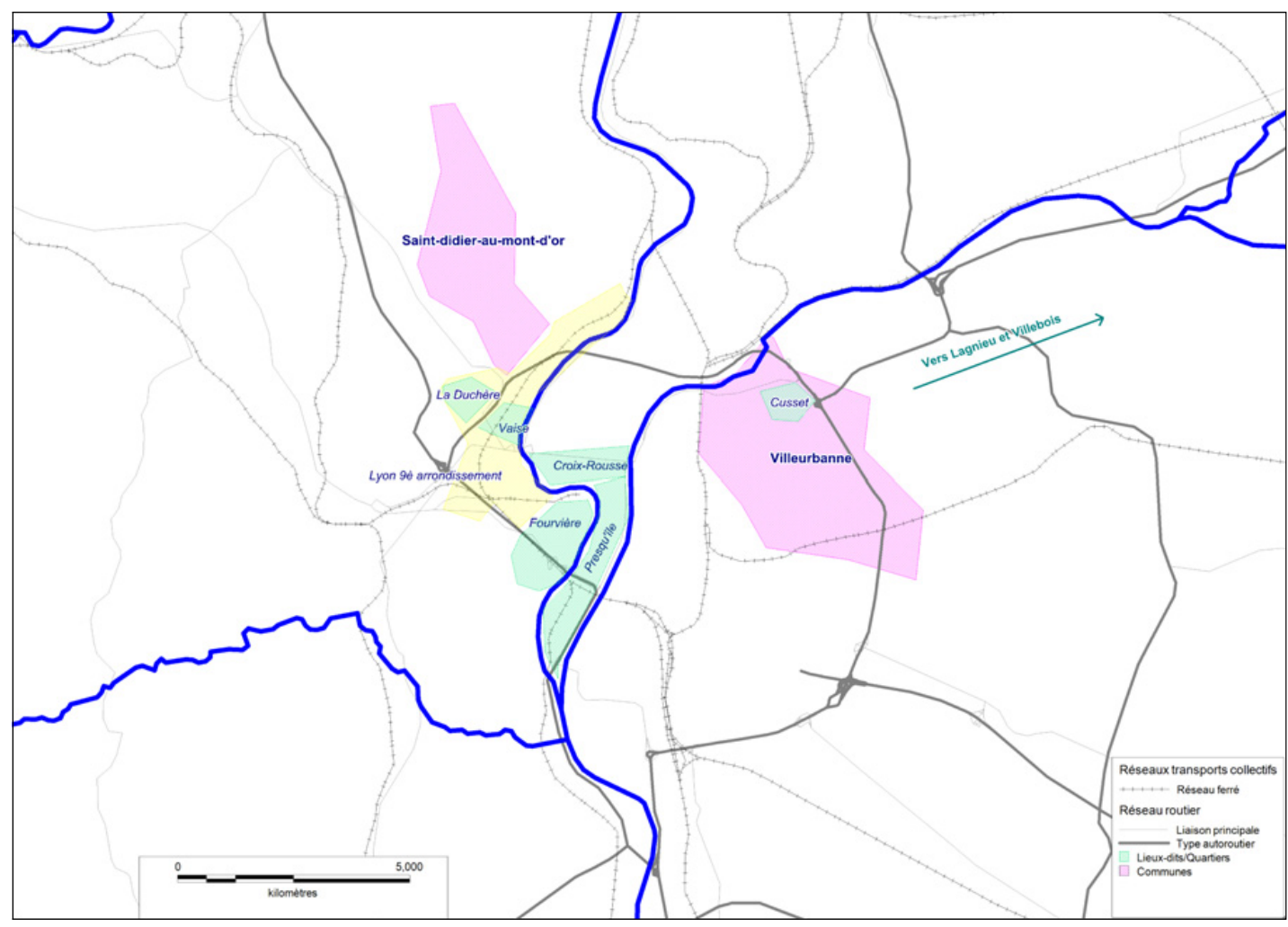

Figure 2. Métropole lyonnaise : les toponymes employés.

entrepris un calcul comparatif méthodique des temps d'accès en transports en commun entre chaque maison visitée et les différents lieux de travail où elle est susceptible d'exercer à l'avenir.

La sœur de Brigitte l'a «alerté sur [l'importance de] faire attention en termes de transports. Des fois, on se dit ce n'est pas grave [d'aller habiter dans le périurbain] et finalement ça peut devenir très lourd dans le quotidien et ça a des répercussions ». Brigitte s'est donné une limite : « c'est 20 minutes au niveau des transports en commun par rapport à la Gare Lille Flandre ». Brigitte est documentaliste en université. Si elle travaille actuellement dans le Vieux-Lille, elle envisage un jour d'avoir à se rendre sur le campus de Lille 2 ou Lille 3, « la gare Lille Flandre, c'est vraiment un point central. Après, il reste un quart d'heure / 20 minutes [de marche ou métro]. Donc au total, je me suis fixé 40-45 minutes pour l'instant [de porte à porte]. C'est aussi ça qui a déterminé les quartiers [de prospection] ». Examen des fiches horaires de bus, consultation des fréquences des métros sur Internet, Brigitte entreprend un vaste travail comparatif qu'elle consigne dans un tableau sur son ordinateur et qui l'amène à « découvrir » la meilleure performance des bus à haut niveau de service.

Le rapprochement des localisations relatives n'est qu'une des facettes des comportements écocompatibles potentiellement annonciateurs d'une transition énergétique. Choisir un mode de transport alternatif, énergétiquement plus sobre ou plus optimisé en est une autre. Le rapprochement des logements des lieux de travail que promeut la ville cohérente [14] n'est pas la seule perspective durable qui soit.

Clara et Florent forment un jeune couple qui souhaite vivre dans un quartier qui « reste accessible à pied [depuis la ville-centre], proche des copains, proche des parents... ». Cette facilité, d'après eux, 


\section{SHS Web of Conferences}

contribue aussi à rendre « les quartiers agréables à vivre ». Ils prospectent donc pour une maison à Lille ou dans la première couronne périphérique lilloise. Pourtant, Florent travaille à Bailleul, et Clara à Armentières. Autant de raisons qui pourraient les pousser à la périurbanisation dans l'ouest de la métropole. Mais ils n'envisagent pas de quitter la ville de Lille. Ils font un choix fort, celui du mode de vie urbain que cette localisation permet. Pétris de valeurs éco-responsables, la localisation de leur futur logement n'est pas jugée optimale d'un point de vue énergétique. Pour autant, Clara et Florent ne cherchent pas à améliorer ces localisations relatives mais optent pour un autre levier d'action : le choix d'un mode de transport alternatif leur permet de rétablir une certaine consonance cognitive. Le couple minimise l'impact (économique autant qu'énergétique) de leur choix résidentiel en préférant entreprendre leurs navettes quotidiennes respectives vers Bailleul et Armentières en covoiturage.

\subsubsection{Le «bon sens » économique}

Il n'y a pas besoin de se revendiquer « militant » ou « écologiste » pour questionner la pertinence de la solution de transport automobile, notamment lorsqu'elle apparaît chère (avant d'être énergivore). À côté de la «figure de l'écolo » il y a la figure du «bon sens » [15]. Il s'opère une diffusion d'un système de valeur qui consiste à s'inquiéter des conséquences à long terme des arbitrages de mobilité ; et la hausse du prix (réel ou psychologique) des carburants y contribue. Or, ce système de valeurs contribue à décourager certaines localisations excentrées ainsi que l'emploi de modes de transport potentiellement énergivores en faisant appel au registre du « bon sens ».

Manuel habite à proximité de Cambrai alors qu'il travaille dans une zone d'activité de la périphérie lilloise. S'il fait régulièrement du covoiturage avec une collègue, c'est que le trajet lui revient cher. Durablement contraint à des navettes domicile-travail importantes, Manuel fait régulièrement la route avec une collègue, en covoiturage. " [J'y vais] les trois quarts du temps en covoiturage, avec une collègue(...). Au départ, [c'est] en discutant, on s'est rendu compte qu'on n'était pas loin. On a continué parce qu'on s'entend bien ». Manuel ajoute : «C'est 5,20€ le péage [à l'aller et au retour], ensuite c'est $120 \mathrm{~km}$ aller-retour ». Or, sa localisation résidentielle n'est pas négociable : « Pour moi c'était un... non-choix » dit-il. Son amie « est patronne d'un bar : c'est son argent c'est son gain, c'est sa clientèle. Et elle est très attachée à l'endroit où elle a acheté, qui est l'endroit où elle est née ». Sans ambiguïté, Manuel pratique le covoiturage pour des raisons économique et non écologique. Par le passé, il a eu la chance de bénéficier d'une grande proximité entre domicile et lieu de travail. Il dit : «Mon lieu de travail était dans la même commune (...) Je mettais 3 minutes [pour y aller] ». Pourtant, Manuel prenait la voiture.

\subsubsection{L' « évidence » qui s'impose au pauvre}

Troisième forme de comportement éco-compatible : la frugalité. Loin de la «tentation ascétique à la décroissance » [3, 16] prônée par certains mouvements écologistes et théoriciens de la retraite [17], un certain nombre de personnes interrogées, parmi celles qui entrent le plus dans la catégorie sociale des petits-moyens, disent faire des économies d'énergie parce qu'elles y sont acculées. À l'instar de familles qui stoppent le chauffage en plein hiver, nous rencontrons des familles qui disent restreindre leurs occasions de mobilité.

Émilie redoute de se laisser séduire par une maison au point de sous-estimer les questions d'accessibilité sous-jacentes pour des raisons économiques, elle craint d'en arriver à dire : « Ah ben je l'aime bien cette maison, mais ah, merde, elle est un peu loin ».

Christiane et Jacques sont intermittents du spectacle à Lyon. Ils vivent dans une maison vétuste, mal isolée et difficile à chauffer, meublée avec des objets de récupération. Ils aimeraient accéder à la propriété mais au fil de leurs prospections, réalisent que le projet est compromis. Leur renonciation 
s'explique en grande partie par le poids économique de la dépense énergétique :

Christiane : «Il faut être au moins à $60 \mathrm{~km}$ pour trouver des choses abordables. (...) Tu achètes un truc pas cher [mais] si ça te coûte le double en transport... ».

Les pratiques éco-compatibles ne s'inscrivent pas toutes, loin s'en faut, dans un système de valeurs revendiquant ou s'appropriant des objectifs de transition énergétique qui dépassent très largement les tribulations quotidiennes de nos interlocuteurs. Interpréter une baisse de mobilité dans ces conditions comme une évolution «allant dans le bon sens » peut même être un contre-sens. Il convient d'être particulièrement prudent avant de prétendre «voir » l'amorce d'une transition énergétique dans l'évolution des comportements de déplacement et les choix de localisation.

\subsection{Une transition qui n'a rien de progressif : des intentions éco-responsables contrariées}

Trois récits nous font entrer dans des projets familiaux qui formulent ou testent l'intention d'un abandon de la voiture individuelle comme principal mode de déplacement sur les navettes domicile-travail. Il est intéressant de comprendre comment ces projets allant dans le sens d'une mobilité plus « durable »-au sens où ils sont potentiellement vecteurs d'une plus grande sobriété énergétique - ont finalement avorté.

\subsubsection{Premier récit}

Lors d'une première entrevue, Pascal et Béatrice envisagent leur futur logement en ces termes :

Béatrice : «Il faut que ce soit dans l'agglomération lyonnaise. On n'a pas de voiture et on n'a pas l'intention d'en acheter. C'est soit en métro, soit pas trop loin à pied, soit en vélo... (...) Je ne vois pas l'intérêt d'acheter une voiture en étant à Lyon. (...) Ça fait deux ans que je n'ai plus de voiture (...) Et de toute façon Pascal n'a pas le permis ».

Le refus d'utiliser la voiture est largement motivé et le discours argumentatif élaboré :

Béatrice : «Pendant deux ans j' avais une voiture. On vivait dans les pentes de la Croix-Rousse ; c'était juste ingérable à garer à chaque fois. À côté de ça tu payes l'assurance, tu paies le parking tu paies... ça te coûte plus cher que de ne pas avoir de voiture. En plus, comme tu ne la prends pas tous les jours parce que tu prends ton abonnement TCL au final, ça sert juste à rien. (...) Et puis au pire, si c'est une fois de temps en temps, il y a toujours la location».

Quelques mois plus tard, lorsque Pascal et Béatrice sont à nouveau interviewés sur leur projet immobilier, le discours est d'une teneur différente. Le projet d'achat s'éloigne avec la perspective imminente d'un départ à l'étranger mais aussi, eu égard aux niveaux des prix du marché immobilier lyonnais.

Béatrice : « [Après le retour du Canada, dans 1 an et demi] peut être qu'on élargira un petit peu notre vue sur d'autres quartiers, un petit peu plus excentrés. Là c'est vrai qu'en tant que jeunes qui aimons sortir, l'idéal... pour nous ça aurait été de retourner dans le $1^{\text {er }}$, sur les pentes de la Croix-Rousse ou dans le $5^{\text {ème }}$ ou sur la presqu'ile. Quand on va revenir, dans un an et demi, ce ne sera plus la vision des choses [en raison d'un projet d'enfant] : il ne faut pas qu'il y ait trop de bruit... Enfin tu vois, que ce soit peut-être possible de garer la voiture si on a besoin d'une voiture... On avait vu que l'on ne voulait pas être trop excentré parce que Pascal est cuistot et quand il finit tard, c'est la merde si on est trop loin, où que ce soit. Peut-être que du coup, si on a un appart, une voiture, peut-être qu'il pourra rentrer en voiture et qu'on pourra être plus loin ».

Malgré leurs intentions initiales de délaisser la voiture, Béatrice et Pascal font évoluer leur projet. Cette résignation tient à la réalité du marché immobilier lyonnais mais aussi à l'anticipation d'un passage à une autre étape du cycle de vie : avoir un enfant. 


\section{SHS Web of Conferences}

\subsubsection{Second récit}

C'est aussi sous le signe de la fatalité que Domitille, après avoir quasiment abandonné l'usage de son véhicule, décide d'y revenir. Lorsqu'elle vivait au Luxembourg Domitille était admirative du système de transport en commun urbain, tant de son étendue que de sa performance.

Domitille : « Ce que j'adorais, (...) c'était que j'avais tous mes clients sur place. Je rentrais le midi faire la sieste, c'était formidable. Je prenais le bus, j'allais chez mes clients en bus, j'allais travailler à pied ou en bus. Mon boulot il était peut-être à.. . 15 minutes en bus ! Et en plus on me payait ma carte de bus! On ne peut pas rêver mieux ! Donc je ne prenais jamais ma voiture... »

Quand elle est revenue vivre en région lilloise, Domitille « avait regardé » comment se rendre en transport en commun dans la zone d'activité de Templemars où elle travaille, mais doit y renoncer :

Domitille : « il y avait les bus mais c'était... [impensable]. Je faisais du contrôle de gestion, j'avais des horaires débiles. Alors à $22 \mathrm{~h}$ reprendre un bus pour aller chez soi... En plus, à l'époque Templemars n'était pas très bien desservi... ». Résignée, Domitille se reconstruit de nouvelles routines automobiles.

\subsubsection{Troisième récit}

Envisager une évolution des routines de déplacement ne présume pas, non plus, d'une remise en question définitive des équilibres anciens. L'inertie des routines peut parfaitement avoir le dessus :

Françoise habite à Wattrelos. Lorsqu'elle a commencé à travailler à Lille, elle s'y rendait en voiture. Puis un jour, à la faveur de l'invitation de deux collègues, elle tente d'utiliser un parking-relais et de continuer en métro : « Au début quand j'ai commencé, on était à trois [à partir ensemble]. (...) La voiture, je la garais à Croix... c'était question gazole, les bouchons et tout ça ».

Mais l'expérience n'a duré que quelques mois : «Le métro c'est une catastrophe, c'était une hantise pour moi, j'en étais malade. (...) Moi je n'arrive pas! Moi j'aime bien être indépendante, je n'aime pas tout ce qui est transport en commun, je déteste. Je n'aime pas le monde, j'aime bien être tranquille le matin et ne pas être embêtée quoi. (...) Donc ça a duré un ou deux mois. Et après j’ai dit non, je reprends la voiture ».

Françoise essaye de prendre le métro pendant quelques mois et finalement abandonne l'expérience et reprend intégralement ses anciennes habitudes de mobilité, comme s'il n'y avait pas de demimesure possible. À l'échelle des pratiques quotidiennes, la transition énergétique ne semble pas pouvoir s'envisager à moitié, elle n'a rien de progressif.

Certaines familles expriment donc des intentions délibérées et aspirations profondes à une évolution de leurs pratiques de déplacement quotidiennes vers des alternatives moins énergivores ; intentions et aspirations qu'elles disent avoir tenté de mettre en œuvre en vain. On peut imaginer que de tels projets avortés auraient constitué des ferments d'évolutions de comportements si des alternatives plus crédibles et plus performantes à l'autosolisme avaient existé (meilleure qualité de desserte, plus grandes fréquences des transports en commun). Mais en l'état actuel de l'offre de transport alternative à la voiture solo, la réalité oscillerait entre deux états laissant peu de place aux situations transitoires :

A changement radical (porté ou non par des valeurs éco-responsables, nous l'avons vu, mais supposant une certaine abnégation)

A et continuité (avec éventuellement quelques ajustements à la marge, comme nous allons le voir).

$\mathrm{Si}$ les représentations sociales peuvent évoluer lentement, au gré de la récurrence des actualités énergétiques ou des annonces d'un effet sur le climat du réchauffement climatique, les changements de comportements de déplacement, quant à eux, sont souvent concomitants d'une rupture radicale des routines du quotidien. Du point de vue des pratiques, il n'y a guère de progressivité dans le changement. 


\title{
3.4 Des ajustements à la marge dans la continuité
}

Lorsque des ajustements s'imposent (pour des raisons économiques notamment), les accédants à la propriété adaptent leurs pratiques quotidiennes à la marge afin de contrarier le moins possible leurs projets résidentiels et/ou leurs routines.

Donia et Stéphane envisagent leurs parcours résidentiels dans une certaine continuité biographique : lorsqu'arrivent de jeunes enfants, l'appartement à Vaulx-en-Velin est jugé trop exigu et le cadre de vie inapproprié. Le pavillon sis à $50 \mathrm{~km}$ de là, dans le périurbain, du côté de Villebois, semble plus approprié. Et Donia et Stéphane vont compenser leur éloignement par une mobilité accrue : « ça va nous faire un petit peu... galoper, on va dire », notamment pour des rendez-vous médicaux réguliers en ville. Les ajustements envisagés ne concernent ni le choix des modes de transport (la voiture est une évidence) ni les localisations relatives (domicile, travail), ils sont à la marge : jusqu'à maintenant, la fille de Donia faisait du judo mais « je ne sais pas si elle pourra refaire du judo l'année prochaine làbas », craint-elle. Habituellement, Donia et Stéphane, se font « un restaurant, tous les 15 jours », mais «là-bas, on ne pourra peut-être pas !».

Pour Francine non plus, les ajustements ne concernent ni les modes de transport ni les localisations, mais à la marge, il affecte l'agencement temporel des activités par un chaînage plus efficace. Travaillant dans la zone d'activité de Templemars, Francine profite du temps de pause les vendredis, à midi, pour se rendre à l'hypermarché situé à proximité. Elle n'a pas le temps de revenir chez elle pour déposer ses achats. Pour les produits frais, elle utilise le réfrigérateur à disposition là où elle travaille.

Francine dit : « À la base c'est pour les gens qui ramènent leur gamelle à midi. Mais c'est des frigos combinés avec le congélateur au-dessus, qui congèlent très bien. Quand j'achète des surgelés, je reviens avec et il n’y a aucun soucis. Moi je fais carrément mon plein de la semaine ».

Habitant dans le périurbain, Francine économise au passage un aller-retour vers un lieu de commerce pendant le week-end.

\begin{abstract}
Zoë a aussi « constaté qu'avec [son] véhicule ça faisait des frais d'essence inutile » de rentrer manger à domicile à l'heure de midi. «Une fois arrivé, il fallait se presser pour manger, je prenais une pause plus longue que nécessaire, je prenais toujours le minimum mais bon. Parce que le temps de revenir, de repartir ça fait 40 minutes, de manger sur place, se préparer à manger, et lancer une machine par exemple... eh bien les deux heures sont rapidement consommées ». Avec Oscar, son époux, ils ont « décidé que l'heure du midi serait profitable » autrement, « en rentrant une heure plus tôt par exemple ». Et depuis, « je ne rentre plus chez moi pour manger », dit Zoë.
\end{abstract}

Face à l'enchérissement des ressources énergétiques, les ajustements envisagés par les familles ne sont pas des plus visibles. Ils se situeraient à la marge et pour l'heure, s'inscriraient dans la continuité. Autant que possible, les familles tentent de préserver leurs projets de vie inscrits dans des projets de parcours résidentiels (inévitablement associés à des routines de mobilité particulières). À la radicalité d'une relocalisation ou d'un abandon de la voiture individuelle, plusieurs familles préfèrent faire le « dos rond ». Dans ces familles plutôt attentistes, les individus laissent venir les changements à eux. D'ici qu'ils soient contraints à des changements (faire évoluer une localisation et/ou des routines de déplacement), ils font le « dos rond »; ils épuisent la « recette automobile » qui a fait ses preuves jusqu'ici.

\section{Conclusion}

Le CREDOC annonçait en titre de l'un de ses rapports que les Français « avancent à grands pas sur la longue route écologique » [18] ; une avancée qui se confirme par la lente évolution de l'« image des modes de transport » chez les Français et par des aspirations relatives à la mobilité nettement plus sobres d'un point de vue énergétique au cours des dix dernières années [19]. En marge de la 
«crise économique» qui impact la France depuis 2008, le portrait d'un Français partisan d'une « frugalité énergétique choisie » [20] peut désormais être brossé statistiquement. En effet, d'un point de vue culturel, on peut avancer que « la transition énergétique avance par le bas, dans un mouvement à bas bruit qui n'est guère perçu par les organisations nationales » [7].

Nos enquêtes ne concluent pas que les individus interrogés se détournent des problématiques écologiques et une conscience écologique - faute de pensée écologisée [21] - se diffuse progressivement dans toutes les catégories sociales. Toutefois, dès qu'on aborde des changements concrets passant par une évolution des pratiques de mobilité ou des choix de localisation affectant la vie quotidienne et les comportements routiniers, un clivage apparaît entre d'une part des personnes qui envisagent le changement sur un mode radical et abouti (écologistes militants) ou sur le mode de la renonciation (familles paupérisées sans autre choix) et d'autre part, des familles qui font le « dos rond », qui s'accrochent à l'existant et qui continuent à n'envisager l'énergie que dans sa dimension locale et conjoncturelle et dans une perspective souvent égocentrée. La notion de transition semble plutôt mal décrire des faits qui sont tout sauf progressifs.

Les résultats que Stéphane Labranche [13, 22] tire de son enquête quantitative rejoignent les nôtres pour penser que nous assistons en premier lieu à une transition dans les représentations qui produit de la dissonance cognitive : «La réduction de l'écart [entre valeurs et pratiques] passe par la modification du discours, des idées, relative aux pratiques dissonantes, plutôt que par un changement des comportements » [13]. Divers « systèmes de justification » [23] aideraient à supporter cette dissonance. Les valeurs environnementales produiraient un malaise à l'échelle individuelle mais assez rarement un changement mécanique dans les pratiques au quotidien. Nos enquêtes montrent cependant que des tentatives d'évolution de pratiques allant dans le sens d'une mobilité durable existent mais avortent.

Pour remédier à la situation, on ne saurait trop répéter qu'il faut garantir la performance et la complémentarité des modes de transport alternatifs à la voiture solo. Si cela est fait de manière constructive, on pourrait aussi rendre obligatoire la rencontre entre un accédant à la propriété et un conseiller info-énergie préalablement à toute transaction immobilière pour examiner avec lui les différents projets immobiliers qui s'offrent à lui afin de les rendre comparables d'un point de vue énergétique eu égard aux destinations routinières et à des impératifs familiaux et professionnels complexes.

\section{Références}

[1] I. Illitch, Energie et équité (Seuil, Paris, 1973)

[2] D. Bourg, K. Whiteside, Vers une démocratie écologique. Le citoyen, le savant et le politique (Seuil, Paris, 2010)

[3] B. Latour, Politiques de la nature ; comment faire entrer les sciences en démocratie ? (La découverte, Paris, 1999)

[4] B. Latour, Enquête sur les modes d'existence; une anthropologie des modernes (La découverte, Paris, 2012)

[5] G. Dupuy in : P. Lannoy, T. Ramadier (éds.), La mobilité généralisée : formes et valeurs de la mobilité quotidienne (Bruylant-Academia, Louvain-la-Neuve, 2007, 41-45)

[6] R. Hopkins, Manuel de transition : de la dépendance au pétrole à la résilience locale (Éditions Écosociété, Paris, 2010)

[7] C. Mincke, B. Montulet, Pol., 64, (2010) URL : http://politique.eu.org/spip.php? article1075

[8] J. Cacciari, R. Dodier, P. Fournier, G. Gallenga, A. Lamanthe, Métrop. (2014) URL : http:// www . metropolitiques. eu/Observer-latransition-energetique.html

[9] M. Cartier, I. Coutant, O. Masclet, Y. Siblot, La France des "petits-moyens ». Enquête sur la banlieue pavillonnaire (La découverte, Paris, 2008) 
[10] Meissonnier J. in J. Armoogum, T. Guilloux, C. Richer (eds.), Observations et analyses croisées des mobilités (éditions du CERTU, Lyon, à paraître)

[11] X. Desjardins, L. Mettetal, Flux, 89/90, 46-57 (2012)

[12] L. Festinger, A theory of cognitive dissonance (Stanford University Press, Stanford, 1957)

[13] S. Labranche, Vertigo, 11 (2012)

[14] E. Korsu, M.H. Massot, J.P. Orfeuil, Le concept de ville cohérente, penser autrement la proximité (La Documentation française, Paris, 2012)

[15] D. Martouzet (ed.), Le périurbain à l'épreuve des modèles d'habiter - La viabilité périurbaine entre théorie(s) et pratique(s) (Rapport de recherche Périvia PUCA, Université de Tours, 2012)

[16] B. Latour, Nous n'avons jamais été modernes. Essai d'anthropologie symétrique (La Découverte, Paris, 1991)

[17] J. Lovelock, The revenge of Gaïa; Earth's Climate Crisis and the Fate of Humanity (Perseus Books Group, New York, 2007)

[18] R. Bigot, S. Hoibian, Cah. de rech. CREDOC, 272 (2010)

[19] V. Kaufmann, K. Tabaka, N. Louvet, J.M. Guidez, Et si les Français n'avaient plus seulement une voiture dans la tête? Evolution de l'image des modes de transport (Lyon, Edition CERTU, 2010)

[20] N. Siounandan, P. Hébel, J. Colin, Cons. et modes de vie, CREDOC, 266 (2014)

[21] E. Morin, L'an I de l'ère écologique (Tallandier, Paris, 2007)

[22] S. Labranche, Env. Urbain / Urban Envir., 5, 10-23 (2011) URL : http://id.erudit.org/ iderudit/1005874ar

[23] A. Rocci in H. Maksim, S. Vincent, C. Gallez, V. Kaufmann (eds.), L'action publique face à la mobilité (L'Harmattan, Paris, 2010) 